\title{
Fabrication and Investigation of Two-Component Film of 2,5-Diphenyloxazole and Octafluoronaphthalene Exhibiting Tunable Blue/Bluish Violet Fluorescence Based on Low Vacuum Physical Vapor Deposition Method
}

\author{
Xiaoyu Zhai, ${ }^{1}$ Siqi Li, ${ }^{1}$ Yufeng Ding, ${ }^{1}$ Liangliang Pan,, ${ }^{1}$ Hejia Yang, ${ }^{1}$ Bingyin Jiang, ${ }^{2}$ \\ Dongpeng Yan, ${ }^{1,3}$ and Qingyun Meng ${ }^{1}$ \\ ${ }^{1}$ State Key Laboratory of Chemical Resource Engineering, Beijing University of Chemical Technology, Beijing 100029, China \\ ${ }^{2}$ Department of Chemistry, SUNY Stony Brook University, Stony Brook, NY 11794-3400, USA \\ ${ }^{3}$ Key Laboratory of Theoretical and Computational Photochemistry, Ministry of Education, College of Chemistry, \\ Beijing Normal University, Beijing 100875, China
}

\begin{abstract}
Correspondence should be addressed to Bingyin Jiang; jiangbingyin@gmail.com, Dongpeng Yan; yandp@mail.buct.edu.cn, and Qingyun Meng; mqybuct@163.com
\end{abstract}

Received 12 April 2016; Accepted 20 July 2016

Academic Editor: Shu Seki

Copyright (C) 2016 Xiaoyu Zhai et al. This is an open access article distributed under the Creative Commons Attribution License, which permits unrestricted use, distribution, and reproduction in any medium, provided the original work is properly cited.

Organic luminescent materials play an important role in the fields of light-emitting diodes and fluorescent imaging. Moreover, new synthetic approaches towards $\pi$-conjugated molecular systems with high fluorescence quantum efficiency are highly desired. Herein, different 2,5-diphenyloxazole-octafluoronaphthalene (DPO-OFN) films with tunable fluorescence have been prepared by Low Vacuum Physical Vapor Deposition (LVPVD) method. DPO-OFN films showed some changed properties, such as molecular vibration and fluorescence. All films exhibited blue/bluish violet fluorescence and showed blue shift, in comparison with pristine DPO. This work introduced a new method to fabricate two-component molecular materials with tunable blue/bluish violet luminescence properties and provided a new perspective to prepare organic luminescent film materials, layer film materials, cocrystal materials, and cocrystal film materials. Importantly, these materials have potential applications in the fields of next generation of photofunctional materials.

\section{Introduction}

During last few decades, organic solid-state photoactive chromophores have received increasing attention due to their promising optoelectronic applications in the fields of lasers $[1,2]$, waveguides [3-6], sensors [7-11], and light-emitting diodes $[1,12,13]$. Compared with their inorganic counterparts, organic photofunctional materials and molecule-based micro/nanomaterials generally possess advantages such as simple process and easily tunable structures, which facilitate the fabrication of flexible and soft devices [14-17]. However, key scientific and technologic problems, such as simpler preparation technology and shorter preparation period, must be resolved before these photoactive molecules can be employed in practical optoelectronic devices [18]. Thus, setting up effective methods to tune the photophysical properties of an organic material is a prerequisite for developing new generation multiple-color luminescent devices [19-24].

Significantly, multicomponent strategies have also emerged as a new direction for the design and fabrication of molecule-based micro/nanomaterials with tunable composition, crystal structures, and solid-state morphologies and properties. This has also shed new light on the structureperformance relationships for molecular solids at the $\mathrm{micro/nanometer} \mathrm{scale}[25,26]$. From the structure and composition perspectives, crystalline micro/nanoarchitecture of a single molecular component has been widely studied and developed $[27,28]$, whereas the design and construction 
of multicomponent crystalline/layered molecular micro/ nanomaterials remain in infancy [29]. Compared with the development of green/red light-emitting materials, the UV and blue light molecular systems with high fluorescence quantum efficiency are still a challenge [30].

DPO is a well-known UV fluorescent molecule [31, 32] with high photoluminescence quantum yield and intriguing luminescent properties [18]. Two-component cocrystal of DPO and OFN has been synthesized by Yan et al. based on solution process [18]. Up to now, while most studies about multicomponent molecular solids were focusing on crystal growth in liquid phase, crystal growth in gaseous phase had gained little attention. Interestingly, compared with solution process, the latter possessed several advantages such as shorter growth period, simpler synthetic process, and more accurate control over the thickness of multicomponent molecular films. Therefore, LVPVD method is potentially a valuable approach in preparing organic light-emitting film materials for large-scale flat panel display and soft optical device [24] with appropriate substrate and organic photoactive materials. The work, herein, is based on LVPVD method to prepare two-component cocrystal films (DPOOFN) and layer films (DPO-OFN) and study the fluorescence properties.

\section{Experimental Section}

2.1. Reagents and Materials. 2,5-Diphenyloxazole (purity: 99\%, mp: $72-74^{\circ} \mathrm{C}$ ) was purchased from J\&K Scientific Ltd., China, and used without further purification. Octafluoronaphthalene (purity: $96 \%, \mathrm{mp}: 87^{\circ} \mathrm{C}$ ) was purchased from Tianjin Heowns Biochemical Technology Co., Ltd., China, and used without further purification. Microscope slides $(25.4 \mathrm{~mm} \times 76.2 \mathrm{~mm} \times 1 \mathrm{~mm})$ were SAIL BRAND, China. Anhydrous ethanol solution (mass fraction: min. 99.7\%) and acetone (mass fraction: min. 99.5\%) were purchased from Beijing Chemical Works, China, and used without further purification.

\subsection{Preparation of DPO-OFN Cocrystal Films and Layer Films.} Microscope slides were cleaned by ultrasound for 10 minutes in anhydrous ethanol, acetone, and deionized water, respectively. DPO-OFN cocrystal films and layer films were prepared by LVPVD method at high temperature $\left(87^{\circ} \mathrm{C}\right)$. Meanwhile, the environmental pressure was 5 pascals and growth time was 1 minute with a DH2010 Multifunctional Vacuum Experimental Instrument. DPO-OFN cocrystal films $(15 \mu \mathrm{m})$ were prepared by heating pristine DPO and OFN before grinding and after grinding, which have been weighed with a $1: 1$ stoichiometric ratio. DPO-OFN cocrystal film in gaseous phase was prepared by heating DPO-OFN cocrystal in liquid phase which was obtained by slow evaporation of a methanol solution containing solid mixtures of DPO and OFN with 1:1 stoichiometric ratio at first. DPO-OFN layer films were prepared by heating pristine DPO and OFN, respectively, which have been weighed with a $1: 1$ stoichiometric ratio. In other words, DPO was heated at first, and then OFN would be heated. DPO-OFN 10-layer film $(2 \mu \mathrm{m})$ was prepared by

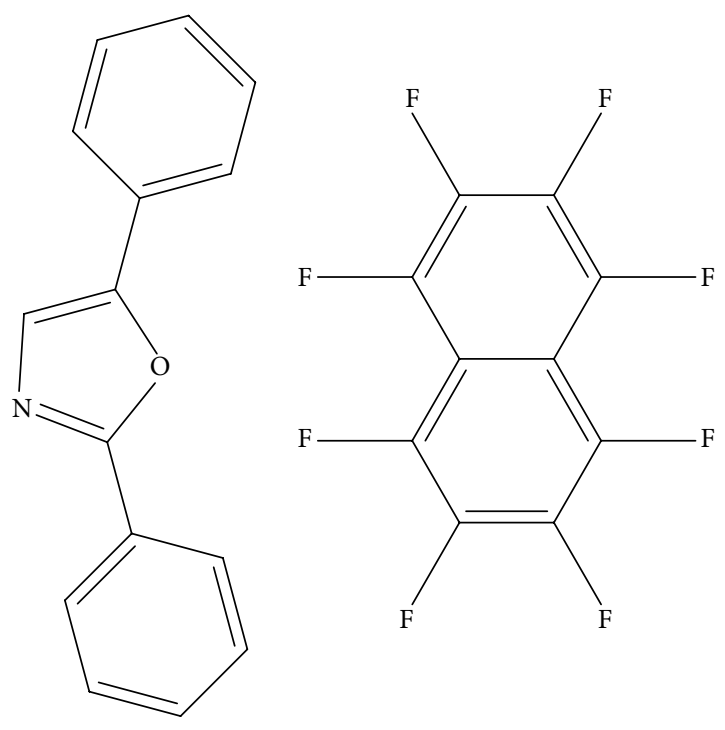

SCHEME 1: Molecular structures of 2,5-diphenyloxazole (left) and octafluoronaphthalene (right).

repeating ten times for the preparation of $200 \mathrm{~nm}$ DPO-OFN layer film.

2.3. Characterization. Raman spectra were recorded on a VERTEX 70v vacuum FT-IR spectrometer. X-ray diffraction (XRD) patterns were recorded using a Bruker D8 Advance X-ray diffractometer under the following conditions: $40 \mathrm{kV}$, $40 \mathrm{~mA}, \mathrm{Cu} \mathrm{K} \alpha$ radiation $(0.154184 \mathrm{~nm})$ with a scanning rate of $10^{\circ} / \mathrm{min}$, and a $2 \theta$ angle ranging from $4^{\circ}$ to $29^{\circ}$ for cocrystal films, $5^{\circ}$ to $31^{\circ}$ for layer films, and $5^{\circ}$ to $27^{\circ}$ for 10-layer film. Surface morphology was recorded on a Hitachi S-4700 field emission scanning electron microscope (SEM), and fluorescence images were obtained on a TCS SP5 Laser Scanning Confocal Microscope with $720 \mathrm{~nm}$ twophoton excitation light. Solid-state fluorescence spectra were recorded on a Hitachi F-7000 FL Spectrophotometer with $270 \mathrm{~nm}$ excitation light. The excitation slit was set to be $2.5 \mathrm{~nm}$ for cocrystal films, layer films, 10-layer film, cocrystal in liquid phase, cocrystal film in gaseous phase, DPO, and OFN, and the emission slit was set to be $2.5 \mathrm{~nm}$ for layer films, while the emission slit was set to be $1.0 \mathrm{~nm}$ for cocrystal films, 10 layer film, cocrystal in liquid phase, cocrystal film in gaseous phase, DPO, and OFN. The photomultiplier voltage was set to be $600 \mathrm{~V}$.

\section{Results and Discussion}

As a representative fluorine-containing compound, OFN (Scheme 1) was chosen as the conformer for DPO. During the preparation of cocrystal films, the probability of encounter between the host molecules and guest molecules needed to be improved to achieve interaction. Significantly, fabrication of DPO-OFN cocrystal films can be achieved by LVPVD method due to similar melting point. Pristine DPO and OFN 


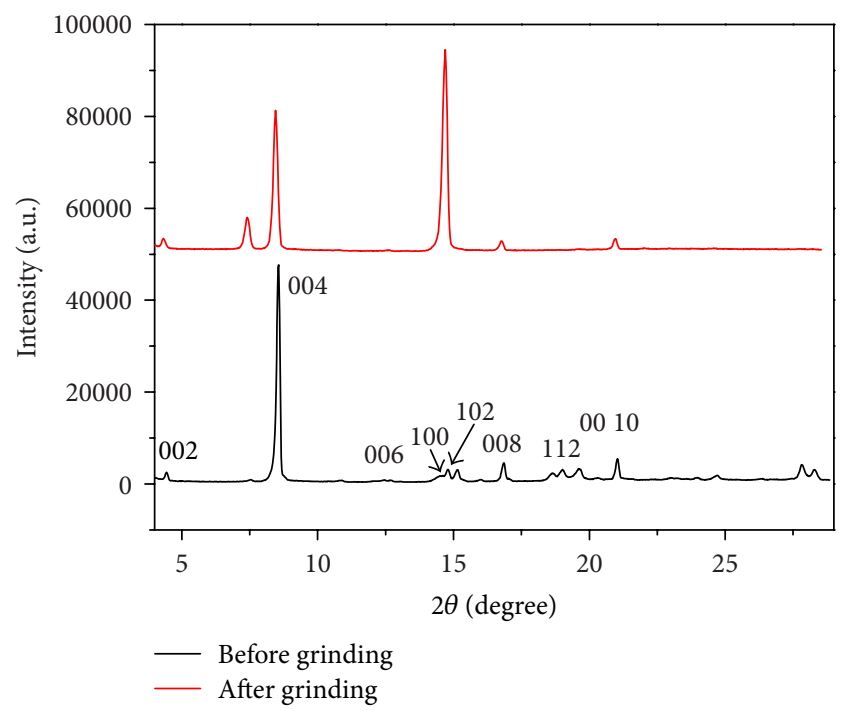

FIGURE 1: XRD pattern for DPO-OFN cocrystal films before and after grinding.

are gasified in a low vacuum environment ( 5 pascal) to interact with each other to fabricate cocrystal films. Interestingly, OFN molecules can isolate the interaction between DPO chromophores, thus suppressing the aggregation of DPO chromophores in the DPO-OFN cocrystal [18]. Therefore, DPO-OFN layer films can be fabricated due to not only their excellent planarity, but also the isolation effect.

3.1. Structural Study on DPO-OFN Cocrystal Films. XRD pattern can help to analyze the structure of a crystal. Figure 1 shows the XRD pattern of DPO-OFN cocrystal films before and after grinding. Compared with the XRD pattern of DPOOFN cocrystal before and after grinding from Yan et al. [18], XRD characteristic peaks of DPO-OFN cocrystal can be observed in Figure 1, which means that DPO-OFN cocrystal has been successfully synthesized by LVPVD method. Meanwhile, Figure 1 shows some diffraction peaks, which cannot be observed in the XRD pattern of DPO-OFN cocrystal from Yan et al. [18], and they have been demonstrated to be the XRD peaks of pristine DPO or OFN due to the noninteracting DPO and OFN on DPO-OFN cocrystal films. Moreover, DPO-OFN cocrystal film before grinding shows better crystal form in comparison with the one of DPO-OFN cocrystal film after grinding by LVPVD method.

Raman spectroscopy was employed to characterize the molecular vibrations within DPO-OFN cocrystal films before and after grinding based on LVPVD method (Figure 2). For pure $\mathrm{DPO}$, the characteristic $\mathrm{C}=\mathrm{C}$ stretching vibration bands of phenyl group appear at 1445, 1478, and $1604 \mathrm{~cm}^{-1}$, and the peak located at $1537 \mathrm{~cm}^{-1}$ can be assigned to the $\mathrm{C}=\mathrm{N}$ vibration of oxazole group [18]. However, for both of $\mathrm{DPO}-\mathrm{OFN}$ cocrystal films before and after grinding, the $\mathrm{C}=\mathrm{C}$ vibration bands at 1445 and $1478 \mathrm{~cm}^{-1}$ underwent a highfrequency shift to $1449 \mathrm{~cm}^{-1}\left(\Delta=4 \mathrm{~cm}^{-1}\right.$ ) and $1482 \mathrm{~cm}^{-1}$ $\left(\Delta=4 \mathrm{~cm}^{-1}\right)$, respectively, indicating that OFN molecules

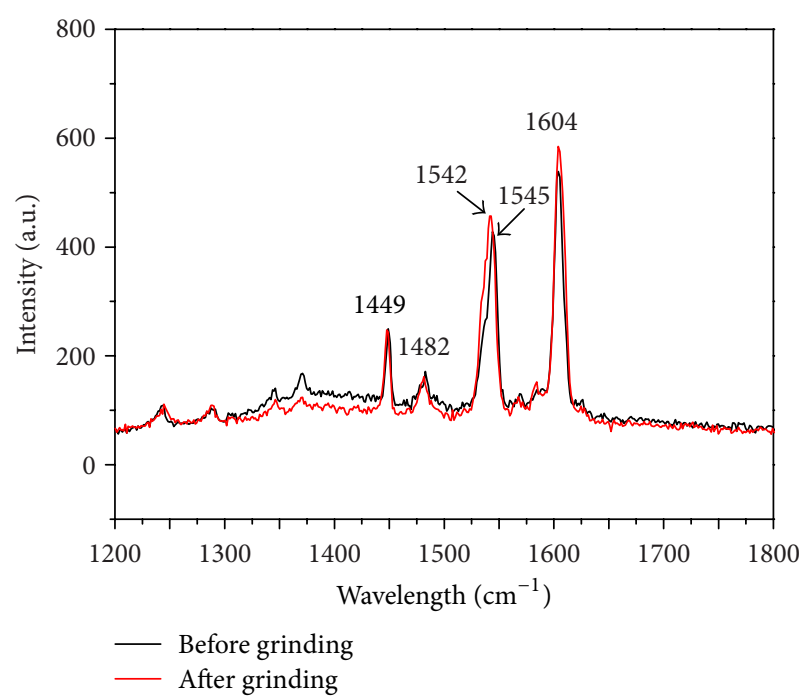

FIGURE 2: Raman spectra for DPO-OFN cocrystal films before and after grinding.

can polarize and delocalize the electronic density of DPO molecules within adjacent layer, which weakened the $\mathrm{C}=\mathrm{C}$ bond in $\mathrm{DPO}$ to some extent, while the $\mathrm{C}=\mathrm{C}$ vibration band at $1604 \mathrm{~cm}^{-1}$ kept no change. Moreover, the $\mathrm{C}=\mathrm{N}$ vibration band at $1537 \mathrm{~cm}^{-1}$ experienced an obvious high-frequency shift towards $1545 \mathrm{~cm}^{-1}\left(\Delta=8 \mathrm{~cm}^{-1}\right)$ and $1542 \mathrm{~cm}^{-1}(\Delta=$ $\left.5 \mathrm{~cm}^{-1}\right)$, respectively, which was indicative of the relative strong halogen bonding interaction between OFN and $\mathrm{N}$ atoms in the oxazole group.

3.2. Surface Morphology and Tunable Fluorescence of DPOOFN Cocrystal Films. Figure 3(a) shows the SEM images for DPO-OFN cocrystal films before and after grinding based on LVPVD method, which show the existence of micro/nanoscale rhabdolith. Importantly, the existence of micro/nanoscale rhabdolith is in agreement with the XRD data in Figure 1, which further demonstrates that DPOOFN cocrystal has been successfully synthesized by LVPVD method. Figure 3(b) shows the fluorescence images of DPOOFN cocrystal films before and after grinding based on LVPVD method. Both films present blue fluorescence, which does not agree with the report of UV fluorescence by Yan et al. [18].

DPO-OFN cocrystal films before and after grinding and DPO-OFN cocrystal film in gaseous phase based on LVPVD method exhibited different fluorescent behaviors from that of DPO-OFN cocrystal by solution process and pristine DPO and OFN. The resulting fluorescence emission spectra were shown in Figure 4. Pure DPO has a maximum emission $\left(\lambda_{\mathrm{em}}{ }^{\max }\right)$ at $392 \mathrm{~nm}\left(S_{1}-S_{0}\right.$ transition) and two shoulder peaks at $377 \mathrm{~nm}(0-0$ vibronic transition) and $412 \mathrm{~nm}(0-$ 2 vibronic transition) [31,32]. In this work, fluorescence emission spectra of pure DPO and OFN show that DPO has two $\lambda_{\mathrm{em}}{ }^{\max }$ at $378 \mathrm{~nm}$ and $391 \mathrm{~nm}$ and one shoulder peak at $412 \mathrm{~nm}$, while OFN has one $\lambda_{\text {em }}{ }^{\max }$ at $353 \mathrm{~nm}$ with $270 \mathrm{~nm}$ excitation light. Compared with pure DPO, the fluorescence 

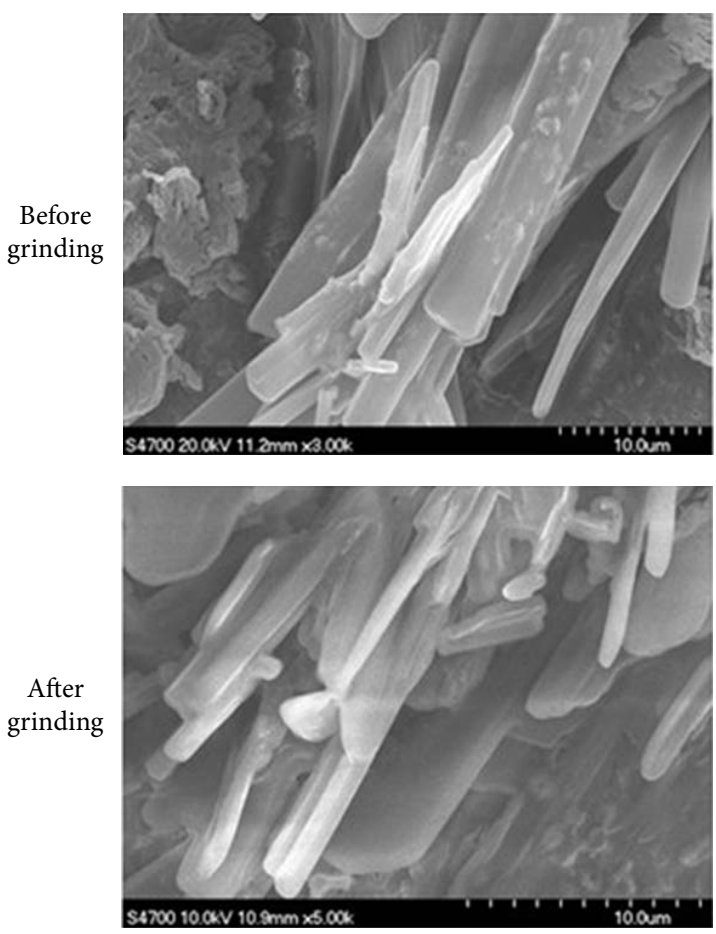

(a)
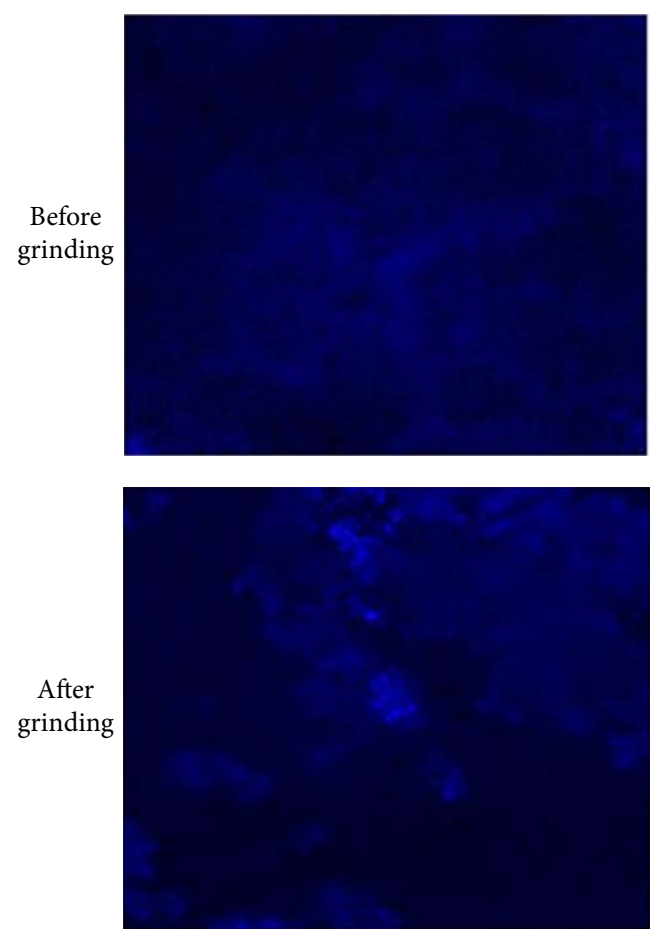

(b)

FIGURE 3: Photographs for DPO-OFN cocrystal films before and after grinding: (a) SEM images; (b) fluorescence images, 94.5-fold (top) and 63-fold (bottom) magnified.

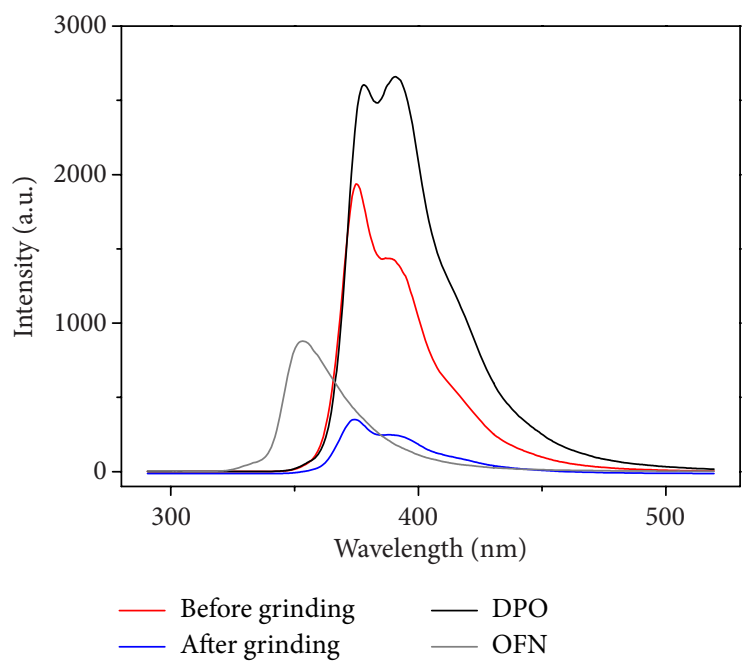

(a)

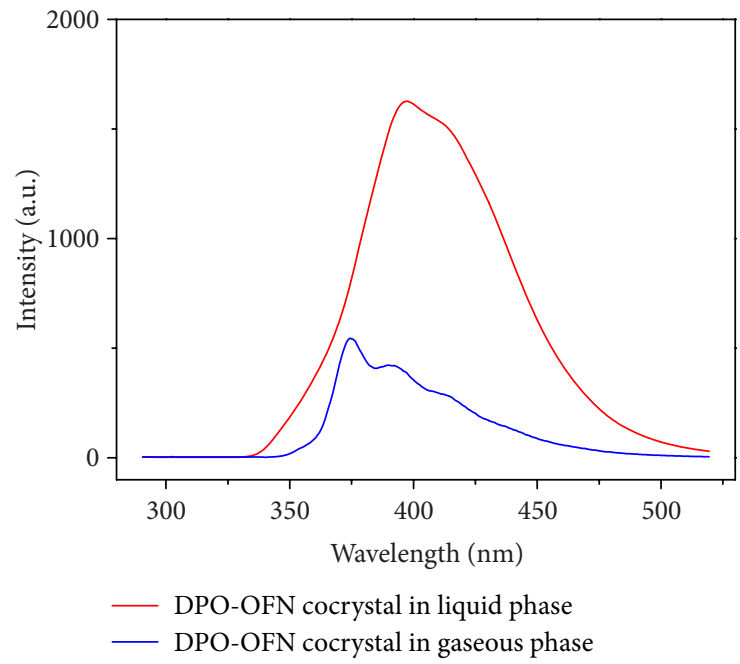

(b)
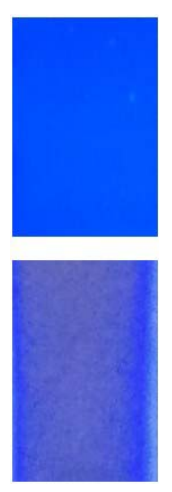

(c)

FIGURE 4: Fluorescence emission spectra and photographs: (a) fluorescence emission spectra for DPO-OFN cocrystal films before and after grinding and pure DPO and OFN; (b) fluorescence emission spectra for DPO-OFN cocrystal in liquid phase and DPO-OFN cocrystal film in gaseous phase; (c) photographs for DPO-OFN cocrystal film before grinding (top) and DPO-OFN cocrystal film in gaseous phase (bottom) under $254 \mathrm{~nm}$ UV.

emission spectra of DPO-OFN cocrystal films before and after grinding both show a blue shift about $3 \mathrm{~nm}$ with $\lambda_{\mathrm{em}}$ max at $375 \mathrm{~nm}$ and $388 \mathrm{~nm}$, respectively, while the shoulder peak at $412 \mathrm{~nm}$ shows no observable shift. In addition, compared with the fluorescence emission spectrum of DPO-OFN cocrystal film before grinding, the one of DPO-OFN cocrystal film after grinding shows no change except for the difference in intensity. Under the same measurement conditions, the emission intensity of DPO-OFN cocrystal film after grinding is lower than the one of the other due to low crystallinity, 


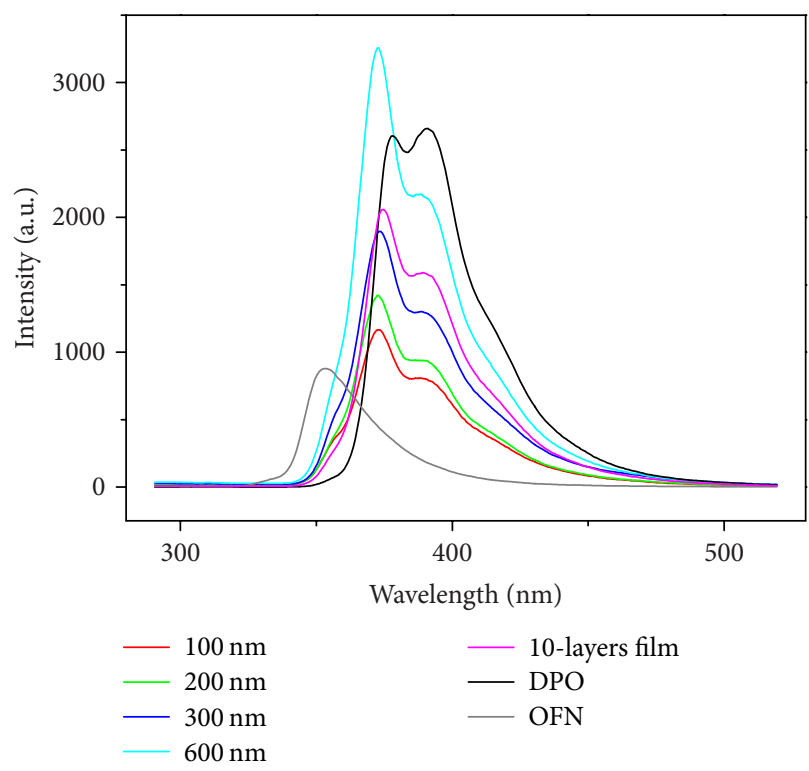

(a)

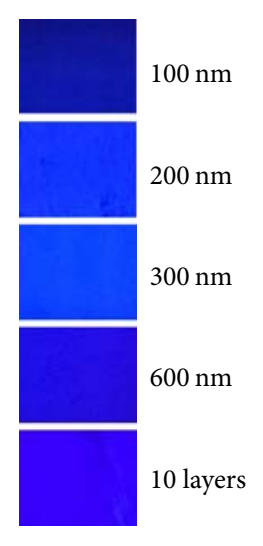

(b)

FIGURE 5: Fluorescence emission spectra and photographs: (a) fluorescence emission spectra for DPO-OFN layer films, DPO-OFN 10-layer film, pure DPO, and OFN; (b) photographs for DPO-OFN layer films and DPO-OFN 10-layer film under $254 \mathrm{~nm}$ UV.

as shown in Figure 1. Fluorescence emission spectrum of DPO-OFN cocrystal in liquid phase shows that DPO-OFN cocrystal has one $\lambda_{\mathrm{em}}{ }^{\max }$ at $397 \mathrm{~nm}$ with a red shift about $6 \mathrm{~nm}$ and one shoulder peak at $412 \mathrm{~nm}$, while $\lambda_{\text {em }}{ }^{\max }$ at $378 \mathrm{~nm}$ of DPO can no longer be observed. Meanwhile, fluorescence emission spectrum of DPO-OFN cocrystal film in gaseous phase shows that the film has one $\lambda_{\mathrm{em}}{ }^{\max }$ at $374 \mathrm{~nm}$ and two shoulder peaks at $390 \mathrm{~nm}$ and $412 \mathrm{~nm}$, with a blue shift about $4 \mathrm{~nm}$ and $1 \mathrm{~nm}$, respectively. Figure 4(c) shows photographs of DPO-OFN cocrystal film (before grinding) and DPO-OFN cocrystal film in gaseous phase under $254 \mathrm{~nm}$ UV. Two films present blue/bluish violet fluorescence.

3.3. Tunable Fluorescence of DPO-OFN Layer Films. DPOOFN layer films with different thickness and DPO-OFN 10 layers film based on LVPVD method exhibit different fluorescent behaviors from that of pristine DPO and OFN, DPOOFN cocrystal in liquid phase, and other films. The resulting fluorescence emission spectra are shown in Figure 5(a). Compared with pure DPO, the fluorescence emission spectra of DPO-OFN layer films show a blue shift about $5 \mathrm{~nm}$ and $3 \mathrm{~nm}$ with $\lambda_{\mathrm{em}}{ }^{\max }$ at $373 \mathrm{~nm}$ and $388 \mathrm{~nm}$, respectively, while the shoulder peak at $412 \mathrm{~nm}$ shows no observable shift. Moreover, the shoulder peak at $353 \mathrm{~nm}$ of DPO-OFN layer films is due to the noninteracting OFN on such films by LVPVD method. In addition, the fluorescence intensity of DPO-OFN layer film intensifies with the increase of thickness of DPO-OFN layer film due to more chromophores. However, the fluorescence spectra of DPO-OFN layer films with different thickness show no change on peak shape and peak position. In order to improve the intensity of layer film, DPO-OFN 10-layer film $(2 \mu \mathrm{m})$ was prepared, where each unit contained $100 \mathrm{~nm}$ thick DPO film and $100 \mathrm{~nm}$ thick OFN film. This structure can isolate the interactions between DPO chromophores, between OFN chromophores, between DPO chromophores and OFN chromophores, and between DPO-OFN cocrystal chromophores, respectively, and thus can suppress the excessive aggregation of chromophores. Significantly, DPOOFN layer film with excellent fluorescence containing color and intensity can be obtained. The fluorescence emission spectrum of DPO-OFN 10-layer film shows a blue shift about $3 \mathrm{~nm}$ and $2 \mathrm{~nm}$ with $\lambda_{\mathrm{em}}{ }^{\max }$ at $375 \mathrm{~nm}$ and $389 \mathrm{~nm}$, respectively, while the shoulder peak at $412 \mathrm{~nm}$ shows no observable shift. Moreover, the shoulder peak at $353 \mathrm{~nm}$ is caused by the noninteracting OFN.

Interestingly, preparation technology will influence the fluorescence of DPO-OFN films. Figure 5(b) shows the photographs of DPO-OFN layer films with different thickness and DPO-OFN 10-layer film under $254 \mathrm{~nm}$ UV. All films exhibit blue fluorescence with different luminance. Moreover, DPO-OFN layer films are slightly dimmer in comparison with DPO-OFN cocrystal film (before grinding) and DPOOFN 10-layer film due to the difference in thickness. DPOOFN cocrystal film before grinding is $15 \mu \mathrm{m}$, and DPO-OFN 10-layer film is $2 \mu \mathrm{m}$, while the thickness of DPO-OFN layer films ranges from $100 \mathrm{~nm}$ to $600 \mathrm{~nm}$.

\subsection{Structural Study and Surface Morphology of DPO-OFN} Layer Films. Considering the fact that $600 \mathrm{~nm}$ DPO-OFN layer film has the maximum thickness among all DPO-OFN layer films in this study, it may exhibit best crystal form among all DPO-OFN layer films. Figure 6(a) shows the SEM image of $600 \mathrm{~nm}$ DPO-OFN layer film, which exhibits no observable crystals like in Figure 3(a). Figure 6(b) shows the XRD pattern of $600 \mathrm{~nm}$ DPO-OFN layer film, where extra peaks are observed, in comparison with the XRD data of 

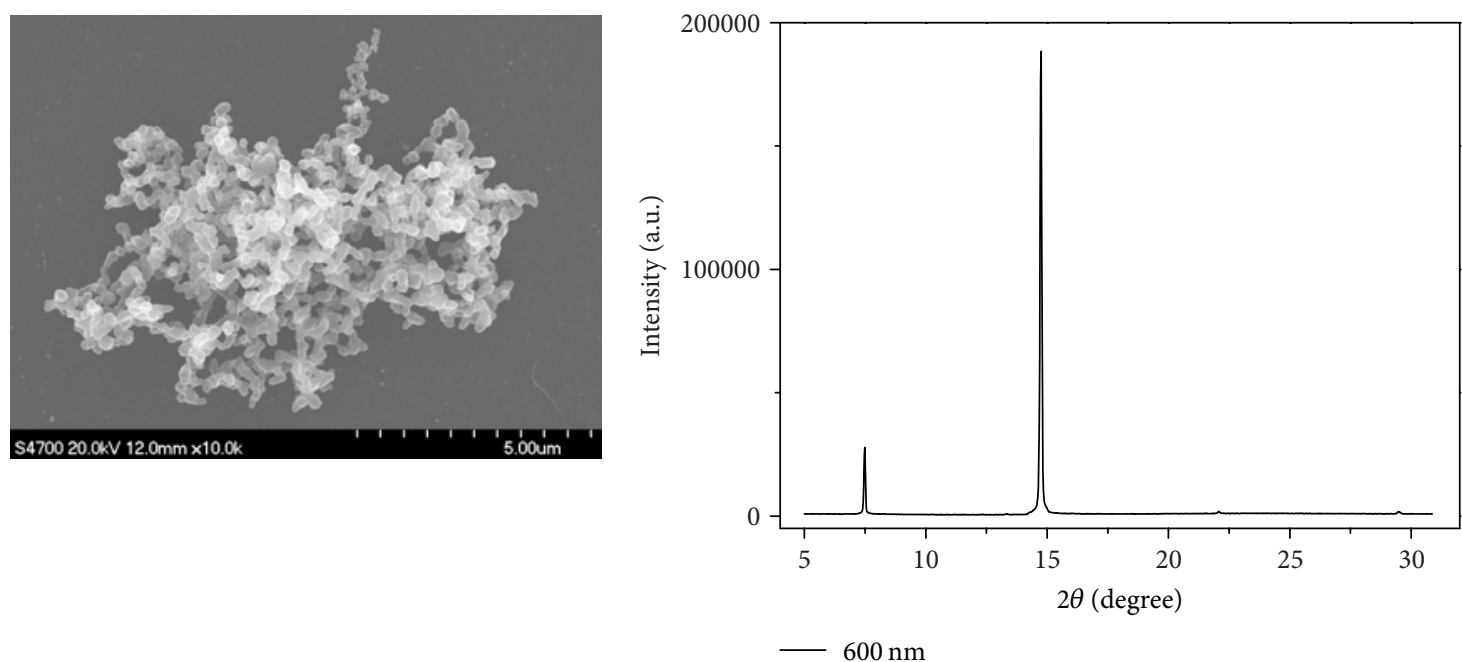

(a)

(b)

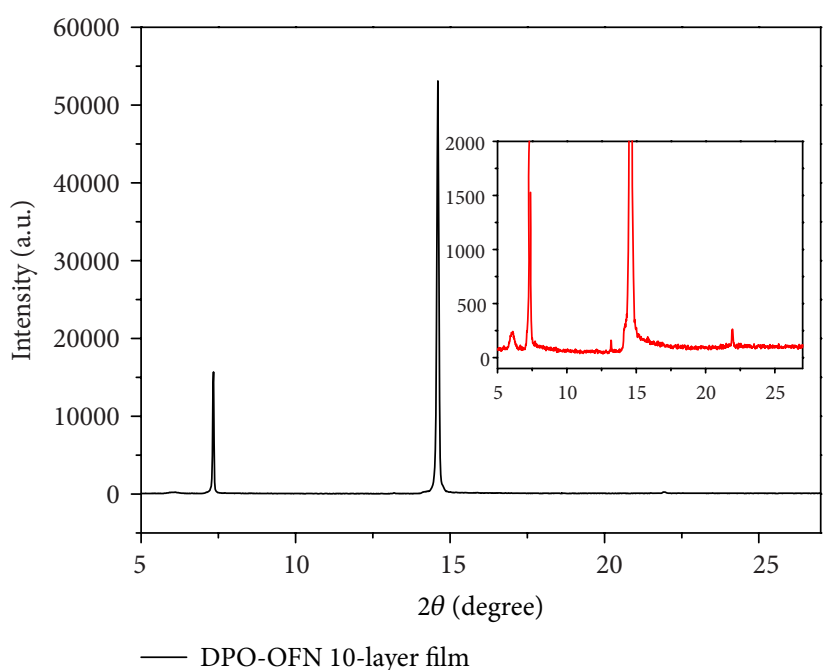

(c)

FIGURE 6: SEM image and XRD patterns: ((a) and (b)) SEM image and XRD pattern for DPO-OFN layer film (600 nm); (c) XRD pattern for DPO-OFN 10-layer film with a partial enlarged profile.

DPO-OFN cocrystal reported by Yan et al. [18]. This can be attributed to the noninteracting DPO or OFN on DPOOFN layer films based on LVPVD method. Figure 6(b) shows worse crystallinity, corresponding to the SEM image. Figure 6(c) shows the XRD pattern of DPO-OFN 10-layer film, where some peaks appear, except the peaks at $6.09^{\circ}$ and $13.18^{\circ}$, which have been demonstrated to be the XRD characteristic peaks of DPO or OFN. The peaks at $6.09^{\circ}$ and $13.18^{\circ}$ have been demonstrated to be two new peaks, which cannot be observed in the XRD pattern of DPO, OFN, or DPO-OFN cocrystal.

\section{Conclusion}

Two-component DPO-OFN films have been fabricated by LVPVD method. The resulting films exhibit some changed properties, such as molecular vibration and fluorescence. Interestingly, all films show novel fluorescence, containing peak shape and peak position, which is different from the one of DPO-OFN cocrystal by solution process, pristine DPO, and OFN. Moreover, DPO-OFN cocrystals have been obtained by LVPVD method with a blue shift. Significantly, LVPVD method was proven to be a capable way to prepare organic luminescent film materials. It will also be effective and provide more perspectives to synthesize new organic luminescent cocrystal materials. In addition, LVPVD method is also anticipated to be useful for fabricating organic lightemitting film materials and next generation of photofunctional materials, which can be utilized in large-scale flat panel display and soft optical device.

\section{Competing Interests}

The authors declare that there are no competing interests regarding the publication of this paper. 


\section{Acknowledgments}

This work was supported by the 973 Program (Grant no. 2014CB932103), the National Natural Science Foundation of China (NSFC), and the 111 Project (Grant no. B07004).

\section{References}

[1] R. H. Friend, R. W. Gymer, A. B. Holmes et al., "Electroluminescence in conjugated polymers," Nature, vol. 397, no. 6715, pp. 121-128, 1999.

[2] S. Tu, S. H. Kim, J. Joseph, D. A. Modarelli, and J. R. Parquette, "Self-assembly of a donor-acceptor nanotube. a strategy to create bicontinuous arrays," Journal of the American Chemical Society, vol. 133, no. 47, pp. 19125-19130, 2011.

[3] H. Yanagi, T. Ohara, and T. Morikawa, "Self-waveguided gainnarrowing of blue light emission from epitaxially oriented $p$ sexiphenyl crystals," Advanced Materials, vol. 13, no. 19, pp. 1452-1455, 2001.

[4] D. O'Carroll, I. Lieberwirth, and G. Redmond, "Microcavity effects and optically pumped lasing in single conjugated polymer nanowires," Nature Nanotechnology, vol. 2, no. 3, pp. 180184, 2007.

[5] Y. Zhao, H. B. Fu, A. D. Peng, Y. Ma, D. B. Xiao, and J. N. Yao, "Low-dimensional nanomaterials based on small organic molecules: preparation and optoelectronic properties," Advanced Materials, vol. 20, no. 15, pp. 2859-2876, 2008.

[6] C. Shi, Z. Guo, Y. Yan et al., "Self-assembly solid-state enhanced red emission of quinolinemalononitrile: optical waveguides and stimuli response," ACS Applied Materials and Interfaces, vol. 5, no. 1, pp. 192-198, 2013.

[7] Y. Che and L. Zang, "Enhanced fluorescence sensing of amine vapor based on ultrathin nanofibers," Chemical Communications, no. 34, pp. 5106-5108, 2009.

[8] Z. Ning, Z. Chen, Q. Zhang et al., "Aggregation-induced emission (AIE)-active starburst triarylamine fluorophores as potential non-doped red emitters for organic light-emitting diodes and $\mathrm{Cl}_{2}$ gas chemodosimeter," Advanced Functional Materials, vol. 17, no. 18, pp. 3799-3807, 2007.

[9] Y. Sagara and T. Kato, "Mechanically induced luminescence changes in molecular assemblies," Nature Chemistry, vol. 1, no. 8, pp. 605-610, 2009.

[10] D. Yan, J. Lu, J. Ma et al., "Layered host-guest materials with reversible piezochromic luminescence," Angewandte ChemieInternational Edition, vol. 50, no. 31, pp. 7037-7040, 2011.

[11] D. Yan, J. Lu, J. Ma, M. Wei, D. G. Evans, and X. Duan, "Reversibly thermochromic, fluorescent ultrathin films with a supramolecular architecture," Angewandte ChemieInternational Edition, vol. 50, no. 3, pp. 720-723, 2011.

[12] C. A. Strassert, C.-H. Chien, M. D. G. Lopez et al., "Lumineszenz eines Platin(II)-Komplexes in gelierenden Nanofasern und elektrolumineszierenden Filmen," Angewandte Chemie, vol. 123, no. 4, pp. 976-980, 2011.

[13] C. A. Strassert, C.-H. Chien, M. D. G. Lopez et al., "Switching on luminescence by the self-assembly of a platinum(II) complex into gelating nanofibers and electroluminescent films," Angewandte Chemie - International Edition, vol. 50, no. 4, pp. 946950, 2011.

[14] A. Vidyasagar, K. Handore, and K. M. Sureshan, "Soft optical devices from self-healing gels formed by oil and sugar-based organogelators," Angewandte Chemie-International Edition, vol. 50, no. 35, pp. 8021-8024, 2011.
[15] A. Vidyasagar, K. Handore, and K. M. Sureshan, "Soft optical devices from self-healing gels formed by oil and sugar-based organogelators," Angewandte Chemie, vol. 123, no. 35, pp. 81718174, 2011.

[16] L. Zang, Y. Che, and J. S. Moore, "One-dimensional selfassembly of planar $\pi$-conjugated molecules: adaptable building blocks for organic nanodevices," Accounts of Chemical Research, vol. 41, no. 12, pp. 1596-1608, 2008.

[17] H. Li, J. Cheng, Y. Zhao et al., "L-Valine methyl ester-containing tetraphenylethene: aggregation-induced emission, aggregationinduced circular dichroism, circularly polarized luminescence, and helical self-assembly," Materials Horizons, vol. 1, no. 5, pp. 518-521, 2014.

[18] D. Yan, H. Yang, Q. Meng, H. Lin, and M. Wei, "Twocomponent molecular materials of 2,5-diphenyloxazole exhibiting tunable ultraviolet/blue polarized emission, pumpenhanced luminescence, and mechanochromic response," Advanced Functional Materials, vol. 24, no. 5, pp. 587-594, 2014.

[19] D. Yan, A. Delori, G. O. Lloyd et al., "A cocrystal strategy to tune the luminescent properties of stilbene-type organic solid-state materials," Angewandte Chemie -International Edition, vol. 50, no. 52, pp. 12483-12486, 2011.

[20] D. J. Wuest, "Molecular solids: co-crystals give light a tune-up," Nature Chemistry, vol. 4, pp. 74-75, 2012.

[21] D. Yan, A. Delori, G. O. Lloyd et al., "Modification of luminescent properties of a coumarin derivative by formation of multicomponent crystals," CrystEngComm, vol. 14, no. 16, pp. 5121$5123,2012$.

[22] D. Yan, D.-K. Bučar, A. Delori et al., "Ultrasound-assisted construction of halogen-bonded nanosized cocrystals that exhibit thermosensitive luminescence," Chemistry - A European Journal, vol. 19, no. 25, pp. 8213-8219, 2013.

[23] D. Yan, B. Patel, A. Delori, W. Jones, and X. Duan, "The formation of hydrogen-bond facilitated salts with tunable optical properties: an experimental and theoretical study of 2,4,5triphenylimidazole," Crystal Growth and Design, vol. 13, no. 1, pp. 333-340, 2013.

[24] D. Yan and D. G. Evans, "Molecular crystalline materials with tunable luminescent properties: from polymorphs to multicomponent solids," Materials Horizons, vol. 1, no. 1, pp. 46-57, 2014.

[25] A. L. Briseno, S. C. B. Mannsfeld, S. A. Jenekhe, Z. Bao, and Y. Xia, "Introducing organic nanowire transistors," Materials Today, vol. 11, no. 4, pp. 38-47, 2008.

[26] J. Huang, S. Virji, B. H. Weiller, and R. B. Kaner, "Nanostructured polyaniline sensors," Chemistry -A European Journal, vol. 10, no. 6, pp. 1314-1319, 2004.

[27] Y. S. Zhao, C. Di, W. Yang, G. Yu, Y. Liu, and J. Yao, "Photoluminescence and electroluminescence from tris(8hydroxyquinoline)aluminum nanowires prepared by adsorbent-assisted physical vapor deposition," Advanced Functional Materials, vol. 16, no. 15, pp. 1985-1991, 2006.

[28] B.-K. An, S. H. Gihm, J. W. Chung, C. R. Park, S.-K. Kwon, and S. Y. Park, "Color-tuned highly fluorescent organic nanowires/nanofabrics: easy massive fabrication and molecular structural origin," Journal of the American Chemical Society, vol. 131, no. 11, pp. 3950-3957, 2009.

[29] D. Yan, "Micro-/nanostructured multicomponent molecular materials: design, assembly, and functionality," Chemistry - $A$ European Journal, vol. 21, no. 13, pp. 4880-4896, 2015. 
[30] M. S. Wong, C. Bosshard, and P. Güter, "Crystal engineering of molecular NLO materials," Advanced Materials, vol. 9, no. 10, pp. 837-842, 1997.

[31] Y. Lei, Q. Liao, H. Fu, and J. Yao, “Orange-blue-orange triblock one-dimensional heterostructures of organic microrods for white-light emission," Journal of the American Chemical Society, vol. 132, no. 6, pp. 1742-1743, 2010.

[32] O. Braem, T. J. Penfold, A. Cannizzo, and M. Chergui, "A femtosecond fluorescence study of vibrational relaxation and cooling dynamics of UV dyes," Physical Chemistry Chemical Physics, vol. 14, no. 10, pp. 3513-3519, 2012. 

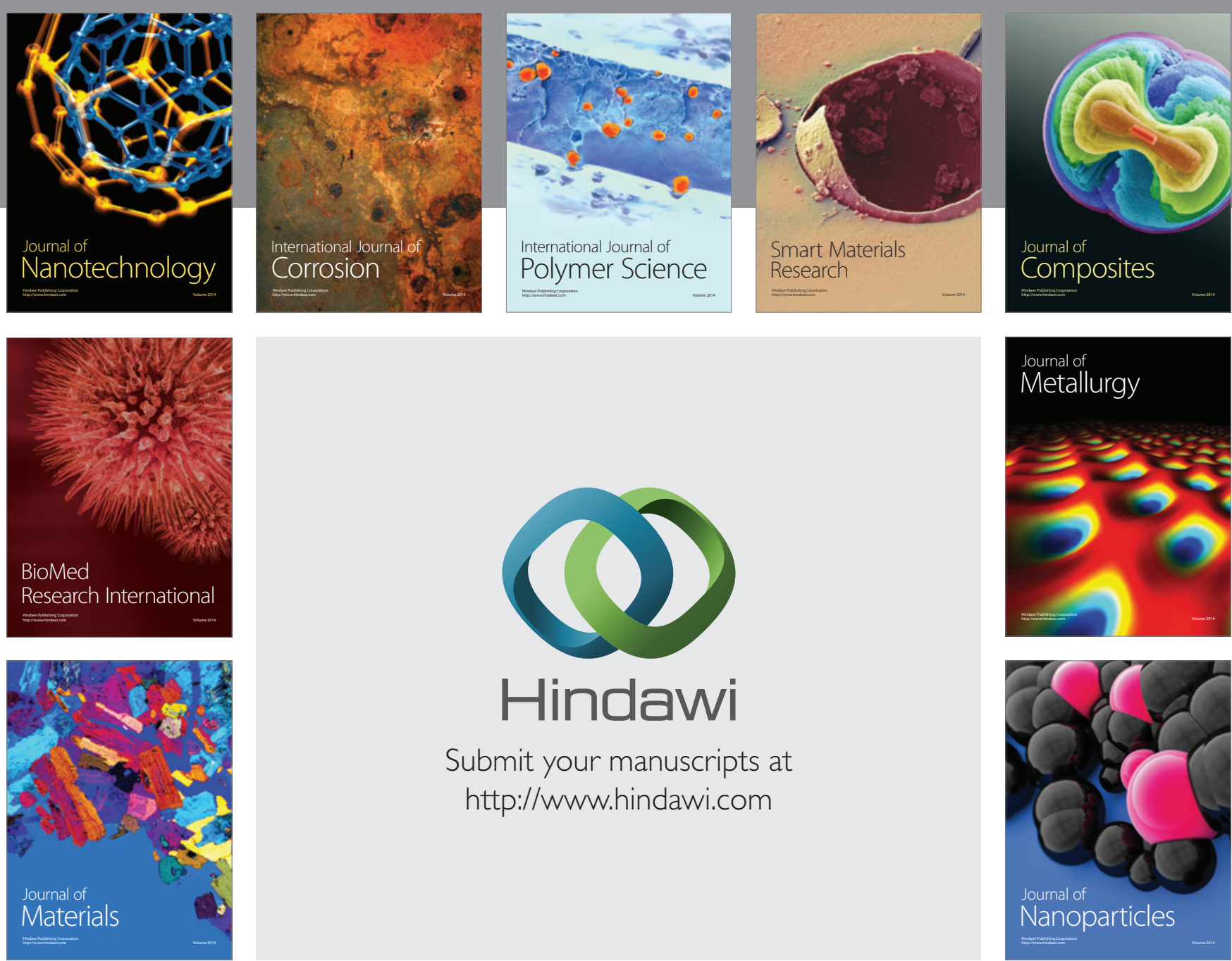

\section{Hindawi}

Submit your manuscripts at

http://www.hindawi.com

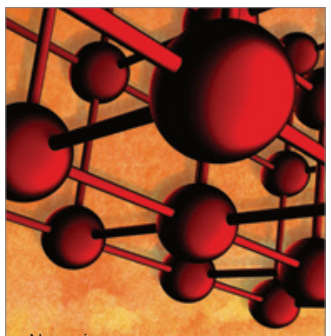

Materials Science and Engineering
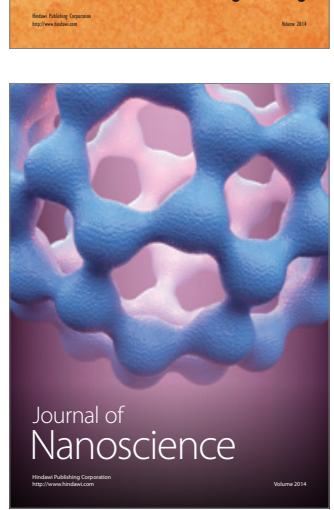
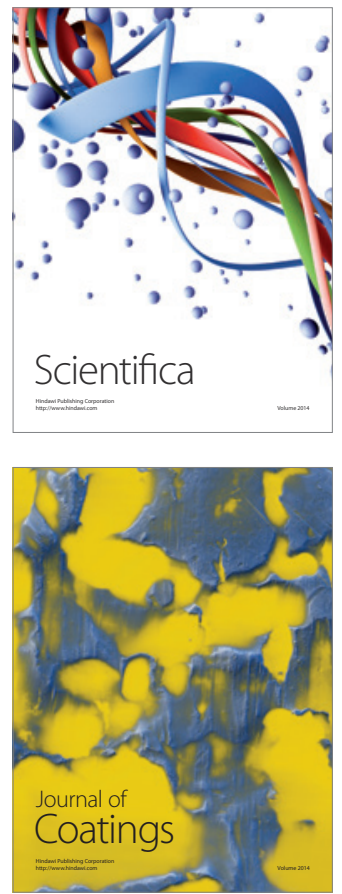
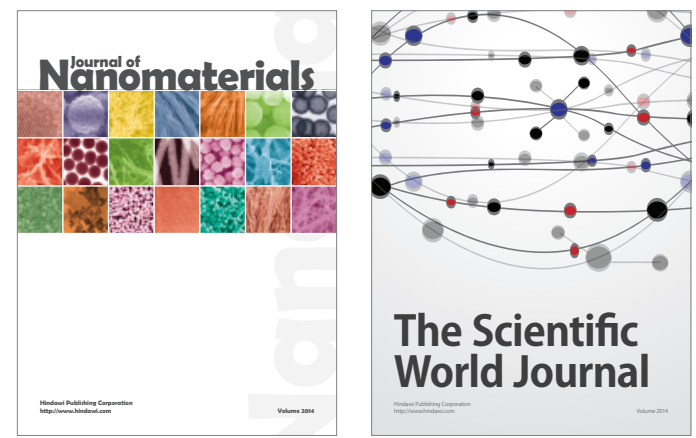

The Scientific World Journal
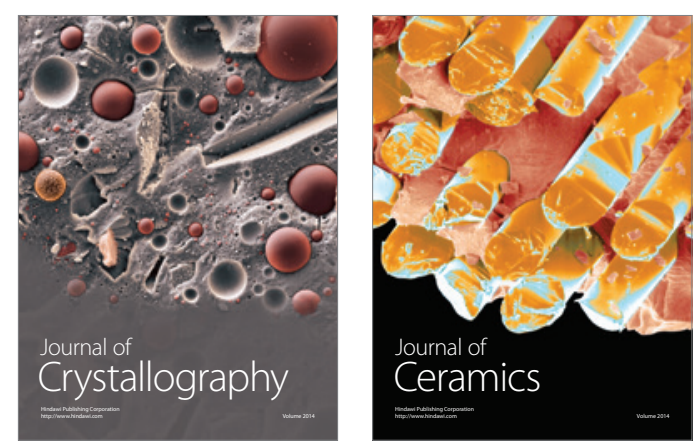
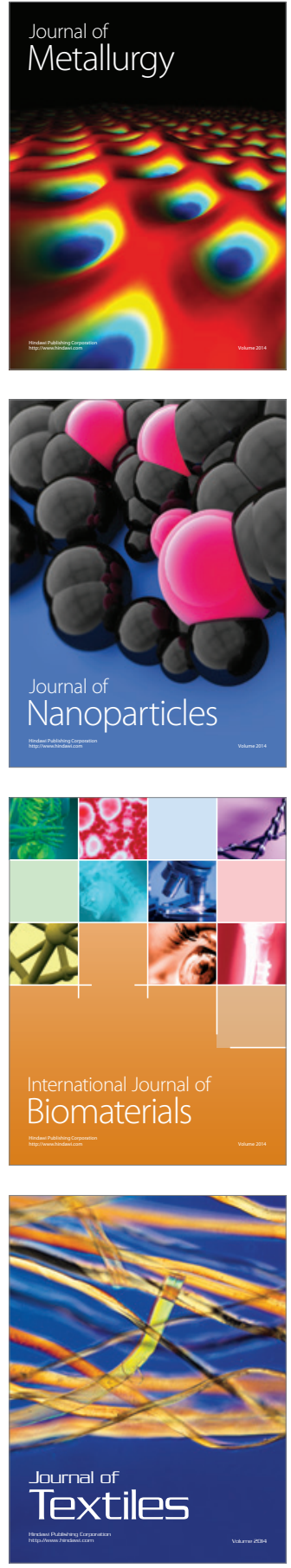